\title{
Design and Analysis of a PLCC Based Home Automation System
}

\author{
M. Gowsalya ${ }^{1}$, M. Barathi Selvaraj ${ }^{2}$, S. E. Murthy ${ }^{2}$, K. Yadhiri $^{2}$ \\ ${ }^{1}$ Embedded System Technologies, Anna University, Chennai, India \\ ${ }^{2}$ Electrical and Electronics Engineering, Knowledge Institute of Technology, Salem, India
}

\section{Email address:}

gowsalyaece90@gmail.com (M. Gowsalya), bseee@kiot.ac.in (M. B. Selvaraj), semeee@kiot.ac.in (S. E. Murthy), kyeee@kiot.ac.in (K. Yadhiri)

\section{To cite this article:}

M. Gowsalya, M. Barathi Selvaraj, S. E. Murthy, K. Yadhiri. Design and Analysis of a PLCC Based Home Automation System. International Journal of Science, Technology and Society. Vol. 3, No. 2, 2015, pp. 36-39. doi: 10.11648/j.ijsts.20150302.11

\begin{abstract}
Traditional metering method is not a convenient for energy measurement and billing. It is more difficult to calculate the bill amount to be paid by a particular user based on the energy consumed and to feed the value to the Electricity Board. This paper aims a new approach for data security and transmission of data using Power Line Carrier Communication (PLCC). This system has an immediate access to the consumer residence in an area with the central board using PLCC. The ARM based system continuously monitors and records the Energy consumption. The smart meter in each residence is connected by central board through the power line. The smart meter calculates the amount to be paid according to the units consumed and also it notify the status of the load connected with it. With help of PLCC the data can transceived from the individual appliances. The data are energy consumption, cost and usage time. The data were transferred from smart meter to knowledge base. This system has the advantages of doing Energy auditing in an area and to find out the losses in the distribution system. LabVIEW software is used to view the output of the individual appliances.
\end{abstract}

Keywords: ARM, PLCC, Lab VIEW

\section{Introduction}

Traditional metering method is not a convenient for energy measurement and billing. This project focused about the status of the home appliance regarding energy consumption and the amount to be paid for the energy consumed. The above mentioned things are displayed in the meter itself. Theenergy consumed by the appliance is monitored by the ARM based system through PLCC.

It is designed for automatic data collection from the appliance and to give intimation to the consumer by displaying on LCD in the meter and control unit. Power consumed by the consumer is monitored by the Electricity Board (EB) through PLCC. The ARM based system records the readings and the live meter reading is displayed in the display.

The ARM automatically takes the responsibility of calculating the bill with the data received from the sensing device, and to monitor the appliance status, unit consumed and amount to be paid. The data send to the smart meter is stored in the backend database of the knowledge base module.
All the data and statics are stored in the central board archives automatically just in the case of any discrepancies.

The system is designed with three modules which are as follows Appliance Module, Consumer Module and Knowledge base Module in the block. These modules show the status, unit consumed and the amount to be paid for the individual appliance. The data sent by the control unit is recorded in the database. The signals are modulated/demodulated and transceivers in all modules.

\section{Literature Survey}

Xi Fang et alsurvey that Power Line Communications (PLC) is a technology for carrying data on a conductor also used for electric power transmission. In the last decades, utility companies around the world have been using PLC for remote metering and load control applications. Although the SG could use many different communications technologies, without a doubt, PLC is the only wired technology that has deployment cost comparable to wireless technologies. Technically, in PLC power electronics are used to manipulate high-voltage waveforms for signal and information oriented 
applications.

A. Vijayaraj et al proposed that in their system has the central EB office to immediate access to all consumer homes in a locality with the help of an RF system. The EB meter present in each house is connected by wireless network with the EB office which periodically gets updates from the meter. The EB office using a backend database calculates the amount to be paid according to the number of units consumed and sends it back to the meter for display and also to the user's mobile phone.

Sajidullah S. Khan et al proposes that this home automation system mainly focused on the design and implementation of a system that control various appliance at home. The design is having Micro controller as its heart and this controller board is connected to the server at the home. Various home appliances are connected to system board with the input/output port. Most of the sensors \& transducers such as temperature, humidity, pressure, are analog. For interfacing these sensors to micro controllers require to convert the analog output of these sensors to digital so that the controller can read it.

Vignesh Set aldescribes that an energy calculation through wireless smart meter using Zigbee is proposed for automatic meter data collection, give intimation through messages displayed on LCD and energy auditing. They discuss about the different hardware techniques for tripping, indicating, intimating the consumers and power monitoring, the Microcontroller based system continuously records the readings and the live meter reading can be sent to the LCD display. The microcontroller automatically takes the responsibility of calculating the bill with the data received from the energy meter, and the tariff provided by the operator and displays the same.

N. Sriskanthan et al proposed that various 'intelligent' appliance such as cellular phones, air-conditioners, home security devices, home theatres, etc. are set to realize the concept of a smart home. They have given rise to a Personal Area Network in home environment, where this entire appliance can be interconnected and monitored using a single controller. Busy families and individuals with physical limitation represent an attractive market for home automation and networking. A wireless home network that does not incur additional costs of wiring would be desirable.

\section{Methodology}

\subsection{Existing System}

In our nation, human beings are involved in obtaining readings from the energy meters, which may cause error. In the existing system, consumers havegone to the EB office to pay bills. The readings are obtained from the analog or digital meter in the consumer residence and the employee from EB calculates the bill which to be paid by the consumer.

The disadvantage lies in bill generation. Here the EB Employee is going to each and every house to take the reading and the bill is generated. This process will take lot of time and laborious task.

The unit consumed by individual appliance and the status of the appliance is unknown. Immediate access to the consumer residence and updated status of the appliance is not possible. Power wastage is not identified.

\subsection{Proposed System}

The proposed system overcomes the above mentioned difficulties by moderating the existing meter with smart meter and by modifying the appliance as a smart one. Each and every appliance can be monitored and controlled with the help of the control unit. The control unit sends the modulated data to the smart meter through wire/wireless communication. The smart meter demodulates the data received and stores the data for analyzing and calculation.

The ARM processor inside the smart meter calculates the energy consumed by the each appliance and stores the data. The smart meter transmits the data to the database every hour. If the consumer wants the status of the appliance, the user can fetch the data from the knowledge base through internet. He can also get the data related to the amount to be paid from the Electricity Board. The consumer can control the appliance by remote.

Smart meter measure the total energy used in the same way as a traditional meter, but they can also inform us when we have used it and how much cost. Additionally, they have a communication capability that allows this data to be displayed on a device inside your home and read remotely by your energy supplier.

CONSUAER MODULE

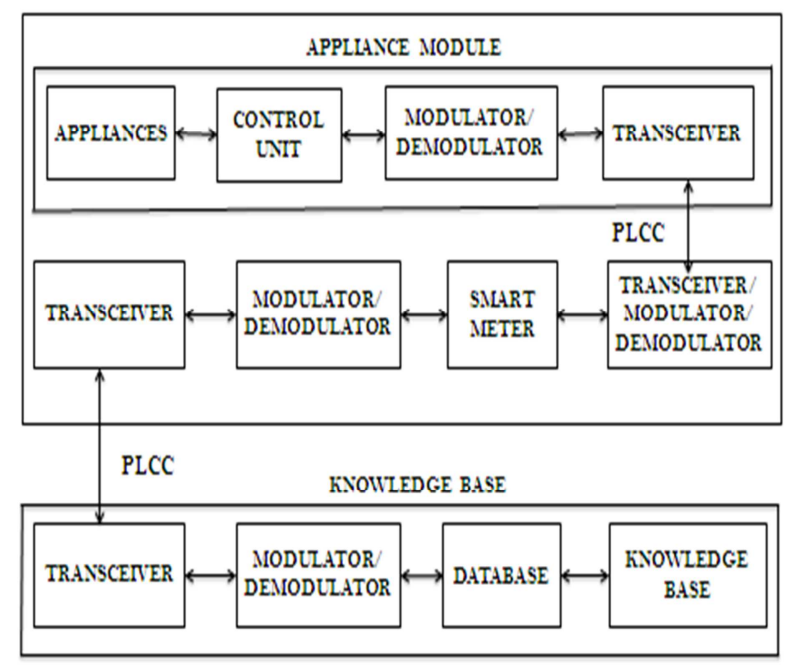

Fig. 3.1. Block diagram of proposed system.

The system mainly operated by ARM processor. In this work, it consists of three modules. They are:

1. Appliance module

2. Consumer module

3. Database module

The Appliance module has the status of the individual appliance and units consumed by individual appliance. The 
data were sending to the control unit and it modulate/demodulate the signal and transceivers through PLCC. Consumer Module has the smart meter and it transceivers the data from the appliance module and was displayed in the meter itself. Database module has the backend database and knowledge base; database has the entire area meter reading record. Knowledge base module has the individual appliance records were show and the update of the data are shown. The signals are modulated/demodulated and transceiver in all modules. These appliances are implemented by using the LabVIEW.

\section{Software Implementation}

The proposed system has implemented LabVIEW which stands for Laboratory Virtual Instrumentation Engineering Workbench, is a graphical computing environment for instrumentation, system design, and signal processing. In my project using Lab view is very easiest and more powerful tools for acquiring, analyzing and presenting real word date.

By using LabVIEW to prototype, design, test, and implement our instrument systems, it can reduce system development time and increase productivity using LabVIEW, can create 32-bit compiled applications that give you the fast execution speeds needed for custom data acquisition, test, Measurement, and control solutions and also can create stand-alone executable mode of function.

\subsection{Flow Chart}

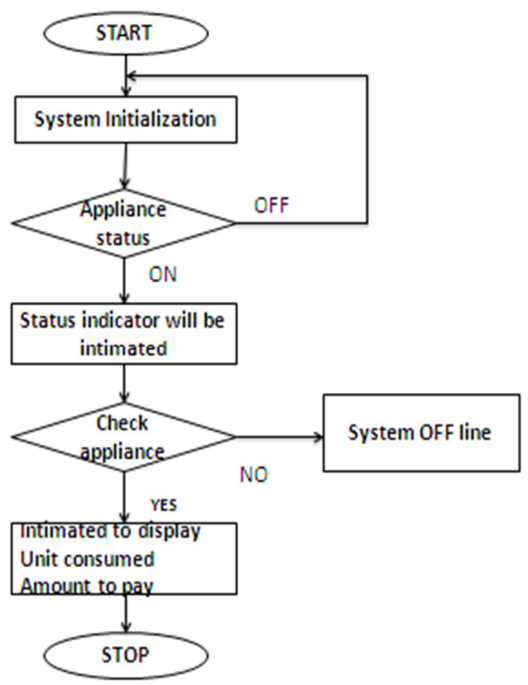

Fig. 4.1. Flow chart.

\subsection{Algorithm}

Step 1: Start the program.

Step 2: Initialization the system. If the appliance is $\mathrm{ON}$ or OFF state.

Step 3: If the appliances in ON state the appliance intimate otherwise again go to step 2 .

Step 4: Check the appliance, if it in ON state it display the unit consumed and amount to pay. In OFF state it displays system OFFLINE.

Step 5: Stop the Program.

\section{Result}

\subsection{LabVIEW Block Diagram and Its Description}

The LabVIEW consist of the front panel and the block diagram. In the block diagram home appliance were represented.

The appliance status like ON/OFF state can be monitored by using the comparator. We have to set the ON/OFF state for the appliance.

If the appliance in ON state the condition is TRUE and indicates green color LED glow in the front panel and display the energy consumed and amount to be pay.

If the appliance in OFF state the condition is FALSE and it indicates red color LED glow in the front panel and display the system offline.

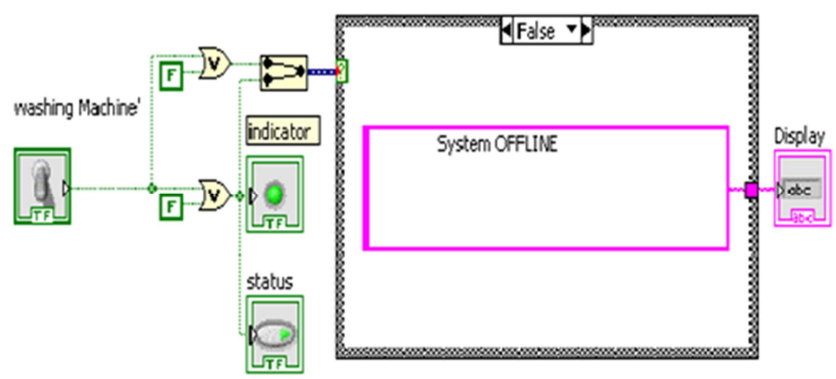

Fig. 5.1. Block diagram in Lab VIEW.

\subsection{Lab VIEW Results}

From the simulation front panel indicates the appliance is in ON/OFF state by LED using Lab VIEW.

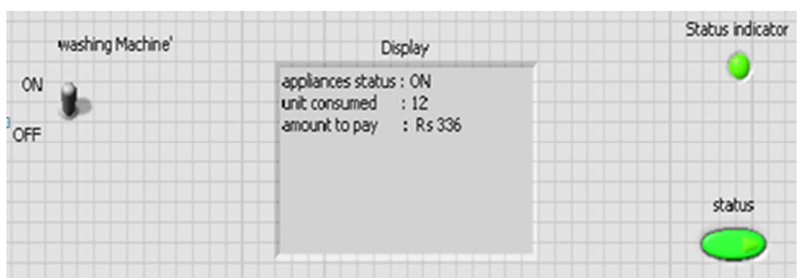

Fig. 5.2. Output ON State in Lab VIEW.

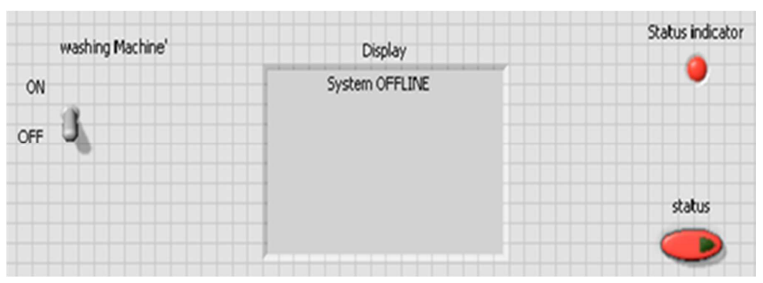

Fig. 5.3. Output OFF State in Lab VIEW.

\subsection{Conclusion}

The ARM automatically takes the responsibility of calculating the bill with the data received from the smart meter, and to monitor the appliance current consumed. The 
data send to the home unit then and there is automatically stored in the Knowledge base module. So, all the data and statistics are stored in the central archives automatically just in case for future references in case of any discrepancies. This system is designed with three modules these modules show the status, unit consumed and the amount to be paid for the individual appliance. The future expansion of the system can be done for Distribution transformer and feeders for monitoring and auditing with substations.

\section{References}

[1] Xi Fang: Smart Grid - The New and Improved Power Grid: A Survey.

[2] A. Vijayaraj: Automated EB billing system using GSM and ad-hoc wireless R outing published at (IJET) 2010.

[3] Sajidullah S. Khan: Home automation system published at /International Journal of Advanced Engineering Technology, April-June, 2011.
[4] Ms.Vignesh: Automatic energy calculation through wireless smart meter Using ZIGBEE published at/International Journal of computer science and Communication networks, 2009.

[5] N.Sriskanthan: Bluetooth based home automation system published at /Microprocessors and Microsystems (2002).

[6] Nagaraju Kommu: Designing of an Automated Power Meter Reading with ZigBee Communication/International Journal of Computer \& Communication Technology, IEEE 2009.

[7] Van Der Weff: A Mobile based home automation system/second International Nov 2005.

[8] InderpreetKaur: Microcontroller Based Home Automation System With Security (IJACSA) International Journal of Advanced Computer Science and Applications, December 2010 .

[9] http://embedsoftdev.com/embedded/Power-line communication.

[10] DevidasThosar: Remote Automatic Meter Reading System with Instant Billing for MSEB/International journal of Emerging Trends in Science and Technology, 2014. 